\title{
Chapter 15 \\ Histochemical Characteristics of Glycoproteins During Rat Palatine Gland Development
}

\author{
Zaki Hakami, Hideki Kitaura, Shiho Honma, Satoshi Wakisaka, \\ and Teruko Takano-Yamamoto
}

\begin{abstract}
Lectin histochemistry has been used to investigate glycosylation modification and glycoprotein expression that occurs during development and under different physiological and pathological conditions. Several lectin histochemical studies have been performed on the palatine gland of different species, which have described the heterogeneity of complex glycoconjugates present in these structures. However, no study has been conducted with regard to the relationship between glycoproteins and palatine gland development in mammals. Therefore, we conducted a study to test the hypothesis that a considerable modification in the expression of carbohydrates occurs in the palatine gland during developmental differentiation and maturation. Histochemical changes of glycoconjugates were observed during prenatal and postnatal development of the rat palatine gland. Qualitative and quantitative differences for the binding of lectins to palatal epithelium sections were determined. All lectins showed general progressive staining during development that was basally extended from the apical cytoplasm of mucous cells. The distribution of glycoproteins during palatine gland development and the heterogeneous distribution of glycoproteins observed between posterior and anterior sides expand our knowledge of the role of salivary glands in oral function. In
\end{abstract}

\footnotetext{
Z. Hakami

Division of Orthodontics and Dentofacial Orthopedics, Department of Translational Medicine,

Tohoku University Graduate School of Dentistry, 4-1 Seiryo-machi, Aoba-ku,

Sendai 980-8575, Japan

Department of Oral Anatomy and Developmental Biology, Osaka University

Graduate School of Dentistry, Osaka 565-0871, Japan

H. Kitaura • T. Takano-Yamamoto $(\bowtie)$

Division of Orthodontics and Dentofacial Orthopedics, Department of Translational Medicine,

Tohoku University Graduate School of Dentistry, 4-1 Seiryo-machi, Aoba-ku,

Sendai 980-8575, Japan

e-mail: t-yamamo@m.tohoku.ac.jp

S. Honma • S. Wakisaka

Department of Oral Anatomy and Developmental Biology, Osaka University

Graduate School of Dentistry, Osaka 565-0871, Japan
} 
this review, we describe and discuss glycohistochemical observations of the developing rat palatine gland.

Keywords Glycoprotein $\bullet$ Lectin $•$ Palatine gland $\bullet$ Rat

\subsection{Introduction}

In humans, the oral cavity and oropharynx are lined by around $600-1,000$ minor salivary glands $[1,2]$. These glands are distributed throughout the mouth except in the gingiva and along the midline and in the anterior part of the hard palate. They consist predominantly of mucous cells that release their secretions through a short ductal system into the oral cavity [3]. Palatine glands are mixed glands of predominantly mucus acini and a few serous demilunes. They are located at the deep termination of secretory units that irregularly grow by pouching [4-6]. In humans they develop from the 11th week of gestation from solid epithelial cord arising from the epithelium lining the soft palate. Thereafter, they undergo lumenization, branching and acinar differentiation [7]. In rats, several thickenings in the palatal epithelium appear at embryonic day 17 (E17). At E18 these have extended as epithelial cords that progressively lumenize and branch to form acini at E20 [8].

Minor salivary glands function semi-continuously throughout the day and night, but only contribute up to $10 \%$ of saliva produced [9]. In addition, they play a major role in the physiological defense mechanism of oral cavity structures by producing of up to two-thirds of mucus and half of the secretory $\operatorname{IgA}$ in the oral cavity [10, 11]. In addition, the most common site of minor salivary gland tumor occurrence is the oral cavity [12-20]. Salivary mucus glycoproteins are numerous and have a tremendous diversity of carbohydrate side chains that are linked to a polypeptide backbone. The advent of lectin histochemistry has allowed such carbohydrate moieties to be characterized. Lectins, which are proteins of plant or animal origin, have been used to visualize glycosylation modification and glycoprotein expression during development or under different physiological and pathological conditions [21-28].

Conventional histochemical methods have revealed that the mucins of rat palatine glands are rich in both acid and neutral glycoconjugates [29], and can incorporate $\left[{ }^{35} \mathrm{~S}\right]$-sulfate [30]. Several lectin histochemical studies have been reported for palatine glands of different species. These emphasize the heterogeneity of the complex glycoconjugates present in these glands, for example $\alpha$-fucose is abundant in mammals, but is scarce or absent in birds [3, 31, 32]. Lectin histochemistry has shown the quantity of glycoproteins to progressively increase during postnatal development of the Magellanic penguin [31]. However, there is a scarcity of information regarding the relationship between glycoproteins and palatine gland development in mammals. Therefore, we hypothesized that a considerable modification in the expression of carbohydrates could occur in the palatine glands during developmental differentiation and maturation, which might coincide with the 
change in diet from milk to solid feeding. In this review, we describe and discuss glycohistochemical observations of the developing rat palatine gland, according to our previous report [33].

\subsection{Palatine Gland During Developmental Differentiation and Maturation}

\subsubsection{Prenatal Stage}

Sprague-Dawley rats were used. The animals were deeply anesthetized with chloral hydrate $(600 \mathrm{mg} / \mathrm{kg}$ body weight, i.p. $)$ and perfused transcardially with $0.02 \mathrm{M}$ phosphate-buffered saline (PBS; pH 7.2) followed by $4 \%$ paraformaldehyde in $0.1 \mathrm{M}$ phosphate buffer (PB; $\mathrm{pH}$ 7.4). For prenatal experiments, the day on which a vaginal plug was recognized was considered as "embryonic day (E) 0". Pregnant mothers at E18, and E20 were sacrificed by an overdose injection of chloral hydrate $(800 \mathrm{mg} / \mathrm{kg})$, and the fetuses were extracted by caesarian surgery. The whole heads were fixed in $4 \%$ paraformaldehyde in $0.1 \mathrm{M} \mathrm{PB}(\mathrm{pH} 7.4)$ for 3 days. All postnatal pups were decalcified with $7.5 \%$ ethylene diamine tetraacetic acid (EDTA) for 1-4 weeks at $4{ }^{\circ} \mathrm{C}$. After decalcification, the head was cut into exact halves along the medial plane. For frozen sections two heads from each group were transferred to PBS containing $20 \%$ sucrose. For paraffin sections, two heads from each group were post-fixed in $4 \%$ paraformaldehyde in $0.1 \mathrm{M} \mathrm{PB}$ (pH 7.4) overnight or for longer. Specimens were then dehydrated by ethanol, cleared in xylene and embedded in paraffin. In accordance with previous reports [7, 8], at E18, gland buds and epithelial cords with a terminal bulb at the distal end were elongated from the epithelial basement membrane (ectoderm) into the stromal connective tissue (mesenchyme). At E20, branching and lumenization had taken place, and immature acini and ducts were formed. We applied lectin histochemistry to frozen sections to avoid false-negative errors. Parasagittal frozen sections were prepared at a thickness of $14 \mu \mathrm{m}$, and mounted on MS-coated glass slides (Matsunami, Osaka, Japan), rinsed with PBS, dried and processed for lectin histochemistry. For the identification of specific carbohydrate residues, tissue sections were incubated for $30 \mathrm{~min}$ with $0.3 \% \mathrm{H}_{2} \mathrm{O}_{2}$ in methanol to block endogenous peroxidase activity. The sections were then incubated for 12-14 h with one of seven different biotinylated lectins, Glycine max (SBA), Dolichos biflorus (DBA), Vicia villosa (VVA), Ulex europaeus (UEA-1), Triticum vulgare (WGA), Succinyl WGA (sucWGA) or Arachis hypogaea (PNA). Sections were then washed three times in PBS, followed by incubation with $A B C$ (Vector Laboratories) for $60 \mathrm{~min}$ and then washed again three times with PBS. The horseradish peroxidase was visualized by incubating slides with $0.05 \mathrm{M}$ Tris- $\mathrm{HCl}$ buffer $(\mathrm{pH} 7.5)$ containing $0.08 \%$ diaminobenzidine and $0.003 \% \mathrm{H}_{2} \mathrm{O}_{2}$. Sections were then lightly counterstained with methyl blue, 
dehydrated and cover slips were mounted using Permount (Fisher, NJ, USA). Sections were examined by light microscopy.

At the prenatal stage, particularly at E18, lectin histochemistry showed considerable variety in the extent or presence of staining among different animals and even among different buds in the same gland. In general, at E18, $\mathrm{N}$-acetylgalactosamine residues visualized by DBA, SBA and VVA showed heterogeneous staining that was negative to weakly positive in the terminal buds. At E21 this staining became progressively more moderate, in the cytoplasm and lumen of ducts, but VVA staining was confined to apical cytoplasm. PNA showed a heterogeneously positive reaction to cell membranes in the epithelial cord but little or no reaction to cells in the terminal buds. At E20, the PNA reaction was located near the lumen. At E18, UEA staining was similar to that of PNA; however, at E20 UEA staining was moderate in the apical cytoplasm and cell membrane as well as on the lumen surface of ducts. WGA reacted strongly at E18, while at E20 staining was moderate on cell membranes and in apical cytoplasm. sucWGA did not show any reaction at E18, but at E20 it stained the cell membrane but not the apical cytoplasm, where secretory granules reside.

In prenatal developmental differentiation, our data showed the importance of terminal sialic acid rather than $\mathrm{N}$-acetylglucosamine, as indicated by intense binding of WGA to the cell membrane and stromal cells and the lack of sucWGA binding [34]. sucWGA labeling appeared largely during postnatal development. Other lectins showed heterogeneous patterns of staining with high affinity of PNA and UEA-1 observed in the epithelial cord at the bud stage. This pattern of reaction is similar to that of developing human labial and lingual minor salivary glands [35]. The varieties in the reactivity of lectins during epithelial budding and bud migration are indicative of a differentiation-dependent alteration in cell surface carbohydrates [36].

\subsubsection{Suckling Stage}

Sprague-Dawley rats aged PN 0-7 were used. We used the same methods for histological analysis and lectin histochemistry as for postnatal animals. The classification of the developmental periods used in this study is based upon the physiological and nutritional stages of animal development [37]. Histological analysis of suckling stage rats showed that the acini of newborns had the general overall appearance of the adult; they contained basophilic nuclei located basally within basal eosinophilic cytoplasm, and pale apical cytoplasm underlined by clear lumen. At day 0 , the secretory units of palatine glands consisted of immature acini and ducts sparsely distributed in the connective tissue, and no extending epithelial cord was observed. The nuclei were round in shape and flattened progressively with age. Gradually with maturation, the glands arranged into lobules, and the number and size of acini increased. Lectin histochemistry at the suckling stage showed that all lectins examined, except PNA, bound to the luminal border and showed generally 
similar reaction patterns. The staining tended to be diffuse or reticular in the apical cytoplasm and apical membrane, as well as in the basolateral membrane of acini in newborns. Moreover, SBA and to a lesser extent PNA showed more positive reactions at supranuclear membranes. The staining by all lectins progressively increased to become moderately distributed. UEA reacted in a similar pattern but was more intense and diffuse, particularly in the acini located on the anterior area of the soft palate where the mother's nipple sits during suckling.

Palatine glands of newborns exhibited the same general overall appearance as those of the adult; therefore, unlike the parotid gland [37], and to a lesser extent the submandibular gland [38], no dramatic histological or morphological changes occurred during postnatal development. Lectin labeling in the newborn was located in the apical portion of the cytoplasm, with slight variation, and with maturation progressively increased and spread out basally corresponding with the progressive enlargement of the apical eosinophilic cytoplasm where secretory granules exist.

\subsubsection{Transitional Stage}

Sprague-Dawley rats aged PN 10-14 were used. In this stage, both suckling and feeding on solid food occur after eruption of teeth and before weaning. The histological sections showed enlargement of the glandular lobules, while the interlobular connective tissue was slightly reduced. Some acini had formed the tubuloacinar and a few scattered serous cells were observed. Histochemically, the distribution of lectin staining remained similar to that in the first week, although the extent of reactions was slightly increased and a granular pattern of staining was noticed in most acini. In addition to mucous cells, UEA showed high affinity to serous cells. sucWGA, however, rarely reacted to serous cells.

\subsubsection{Weaning Stage}

Sprague-Dawley rats aged PN 21-28 were used. Histological analysis showed that the general appearance of the gland remained unchanged at the weaning stage; however, the whole gland at 4 weeks appeared more compact, the eosinophilic cytoplasm of the mucous cells was enlarged and nuclei were further flattened and displaced basally. Serous acinar cells were observed along the soft palate, predominantly in the posterior part. Some were capped in the mucous acini and others were isolated. Lectin histochemistry at the third week showed heterogeneous staining among different animals; however, overall patterns similar to those of the preceding stage were seen for WGA, sucWGA, UEA and PNA. SBA, sucWGA and UEA-1 showed affinity to serous cells, and the reactivity of DBA and VVA was expanded to cover the basal region and supranuclear membrane of some acinar cells. Some cells exhibited a similar pattern to that found in the adult. At the fourth week, the 
lectin staining patterns were mostly similar to those found in the adult, in which reactivity was extended basally from the apical cytoplasm along the lateral surface of acinar cells. DBA staining was strong, covering the entire cytoplasm. Staining for VVA, WGA and UEA was weaker but there was no significant difference between anterior and posterior regions. Surprisingly, the reactivity of PNA and sucWGA was reduced by more than one-third and focal, moderate cytoplasmic staining was observed more in the posterior glands. At the third week, strong, broad distribution of lectin binding was observed; in particular, PNA and sucWGA reached their peaks of reactivity. These additional mucous secretions might be required as a lubricant for both chewing and swallowing solid food and may provide a protective coating for the soft palate [39-41], and are thus consistent with forced weaning.

\subsubsection{Adult Stage}

Sprague-Dawley rats aged PN 42 were used. The histological analysis of the adult stage showed that the thickness of the glandular layer was increased, but the thickness of the posterior portion was clearly smaller than the anterior oral one. Glands appeared more dilated, and the faint apical cytoplasm was enlarged and pressed the spindle-shaped nuclei against the basement membrane. Serous cells with round nuclei and basophilic cytoplasm were observed along the soft palate, mainly in the posterior portion, some of which were isolated while others were capped with mucous cells. Lectin histochemistry at the adult stage showed a dramatic heterogeneity of glycoproteins between the anterior and posterior portions. DBA, VVA and WGA showed high affinity to all mucous cells, but their staining patterns were more broadly and intensely distributed in the posterior portion. The reactivity of SBA, however, was located in the apical cytoplasm in the anterior portion and was more intense and broad in the posterior region. Furthermore, less than one-third of cells showed binding to PNA and sucWGA, most of which were located in the posterior portion. Finally, UEA-1 reacted strongly and was evenly distributed along the palatine gland.

This study showed that the 4 th week was the appropriate time for weaning, when the histochemical distribution of lectins among mucous cells of palatine glands behaved mostly like that of the adult. Our study showed that the mucins secreted from the palatine gland changed in quality and quantity during growth. Moreover, in the adult stage, a spectacular heterogeneous distribution of glycoconjugates was observed in the soft palate between palatine glands located in the anterior and posterior portions; the posterior side was rich in $\mathrm{N}$-acetylglucosamine and galactose compared with the anterior side as demonstrated by its positive reactivity with sucWGA and PNA, respectively. N-acetylgalactosamine was also more abundant in the posterior side as demonstrated by intense and broad distribution of DBA, VVA and SBA. Heterogeneous distribution of glycoconjugates within an organ has been previously described for the kidney of JDS mice; distal tubules showed binding to 
DBA, whereas proximal tubules did not [22]. Also, in the human large bowel, UEA-1 bound to mucous goblet cells proximally but not distally [42]. Generally, glycoproteins secreted by mucous cells contain both O-linked oligosaccharides, which contribute to the protective physiochemical properties of the mucus coat, and $\mathrm{N}$-linked oligosaccharides. In the present study, N-linked oligosaccharides, as indicated by mannose directed ConA lectin binding, were evenly distributed throughout the soft palate. However, dramatic distinguishable differences were revealed between anterior and posterior regions, where O-linked oligosaccharides were highly expressed in the latter as indicated by DBA, VVA, SBA and PNA staining [22]. An apocrine mechanism of secretion by mucous cells in the salivary gland has been excluded [43]; therefore, two reasons may be speculated to explain the abovementioned heterogeneity in the soft palate. First, the epithelium covering the soft palate has fewer layers in the posterior portion, and the thickness of the glandular layer of the palatine gland is obviously smaller, which strikingly correlated with presence of more abundant glycoproteins in that area. This suggests that palatine glands located in the posterior portion, as a functional compensation, produce expanded and elongated mucin by over-secretion of glycoproteins with O-linked oligosaccharides that change the physiochemical properties of the mucin by making it more viscous, with lower solubility and higher elasticity and adhesiveness. This in turn provides additional integrity to the soft palate mucosa from any mechanical or chemical injury $[44,45]$. The second speculation is inspired from the suggestion that serous demilunar and central acinar cells might be the phenotype of a single secretory-cell type [46]. Accordingly, we observed that SBA, PNA and sucWGA sometimes reacted to serous cells in addition to mucous cells during the stages examined, while in the adult samples they showed higher affinity to serous cells of the von Ebner gland. Therefore, the more abundant serous cells in the posterior portion probably could have contributed to the production of glycoproteins and were subsequently expressed by lectin binding in the main acinar cells due to glycosylation continuation of demilunes to main mucous cells.

\subsection{Conclusions}

Lectin histochemistry has the ability of identifying oligosaccharide-specific residues in histological sections and delineating information about the structure of carbohydrate-rich macromolecules. The method is useful for the analysis of palatal gland development. The present observations revealed that glycoprotein distribution during palatine gland development varies with age. This variation in staining properties could be related to the maturation process in the secretory cycle of the palatine mucous glands. Moreover, the heterogeneous distribution of glycoconjugates between posterior and anterior glands, which is probably due to different functional demands, expands our understanding of the role of salivary glands in oral function. Further studies are needed to elaborate further physiochemical and rheological differences. 
Open Access This chapter is distributed under the terms of the Creative Commons Attribution Noncommercial License, which permits any noncommercial use, distribution, and reproduction in any medium, provided the original author(s) and source are credited.

\section{References}

1. Kitagawa K, Hayasaka S, Matsunou H, Nagaki Y. Presumed minor salivary gland secretion in a patient with a history of oral mucous membrane graft. Am J Ophthalmol. 2003;136(2):374-5.

2. Sivarajasingam V, Drummond JR. Measurements of human minor salivary gland secretions from different oral sites. Arch Oral Biol. 1995;40(8):723-9.

3. Hand AR, Pathmanathan D, Field RB. Morphological features of the minor salivary glands. Arch Oral Biol. 1999;44 Suppl 1:S3-S10.

4. Leeson CR, Leeson TS. Fine structure and possible secretory mechanism of rat palatine glands. J Dent Res. 1968;47(4):653-62.

5. Nakamura S, Takahashi S, Wakita M, Morita M. Postnatal growth of the rat palatine gland. Tissue Cell. 2001;33(6):614-20.

6. Srivastava HC, Vyas DC. Postnatal development of rat soft palate. J Anat. 1979;128 (Pt 1):97-105.

7. Nielsen G, Westergaard E. The development of the palatine glands in human foetuses with a crown-rump length of 32-145 mm. Acta Odontol Scand. 1971;29(2):231-50.

8. Shinzato K, Takahashi S, Wakita M, Morita M. Prenatal development of the palatine gland of rats. Tissue Cell. 2004;36(2):115-20.

9. Eliasson L, Carlen A. An update on minor salivary gland secretions. Eur J Oral Sci. 2010;118 (5):435-42.

10. Crawford JM, Taubman MA, Smith DJ. Minor salivary glands as a major source of secretory immunoglobin A in the human oral cavity. Science. 1975;190(4220):1206-9.

11. Dawes C, Wood CM. The contribution of oral minor mucous gland secretions to the volume of whole saliva in man. Arch Oral Biol. 1973;18(3):337-42.

12. Buchner A, Merrell PW, Carpenter WM. Relative frequency of intra-oral minor salivary gland tumors: a study of 380 cases from northern California and comparison to reports from other parts of the world. J Oral Pathol Med. 2007;36(4):207-14.

13. Carrillo JF, Maldonado F, Carrillo LC, Ramirez-Ortega MC, Pizano JG, Melo C, et al. Prognostic factors in patients with minor salivary gland carcinoma of the oral cavity and oropharynx. Head Neck. 2011;33(10):1406-12.

14. Chuiwa H, Sakamoto K, Umeno H, Nakashima T, Suzuki G, Hayafuchi N. Minor salivary gland carcinomas of oral cavity and oropharynx. J Laryngol Otol Suppl. 2009;31:52-7.

15. Hyam DM, Veness MJ, Morgan GJ. Minor salivary gland carcinoma involving the oral cavity or oropharynx. Aust Dent J. 2004;49(1):16-9.

16. Kakarala K, Bhattacharyya N. Survival in oral cavity minor salivary gland carcinoma. Otolaryngol Head Neck Surg. 2010;143(1):122-6.

17. Lee SY, Shin HA, Rho KJ, Chung HJ, Kim SH, Choi EC. Characteristics, management of the neck, and oncological outcomes of malignant minor salivary gland tumours in the oral and sinonasal regions. Br J Oral Maxillofac Surg. 2013;51(7):e142-7.

18. Matsuzaki H, Yanagi Y, Hara M, Katase N, Asaumi J, Hisatomi M, et al. Minor salivary gland tumors in the oral cavity: diagnostic value of dynamic contrast-enhanced MRI. Eur J Radiol. 2012;81(10):2684-91.

19. Spiro RH. Salivary neoplasms: overview of a 35 -year experience with 2,807 patients. Head Neck Surg. 1986;8(3):177-84.

20. Spiro RH, Thaler HT, Hicks WF, Kher UA, Huvos AH, Strong EW. The importance of clinical staging of minor salivary gland carcinoma. Am J Surg. 1991;162(4):330-6. 
21. Rademacher TW, Parekh RB, Dwek RA. Glycobiology. Annu Rev Biochem. 1988;57:785-838.

22. Spicer SS, Schulte BA. Diversity of cell glycoconjugates shown histochemically: a perspective. J Histochem Cytochem. 1992;40(1):1-38.

23. Ferrari MC, Parini R, Di Rocco MD, Radetti G, Beck-Peccoz P, Persani L. Lectin analyses of glycoprotein hormones in patients with congenital disorders of glycosylation. Eur $\mathrm{J}$ Endocrinol. 2001;144(4):409-16.

24. Goodarzi MT, Turner GA. A lectin method for investigating the glycosylation of nanogram amounts of purified glycoprotein. Glycoconj J. 1997;14(4):493-6.

25. Hayes CA, Doohan R, Kirkley D, Leister K, Harhen B, Savage AV, et al. Cross validation of liquid chromatography-mass spectrometry and lectin array for monitoring glycosylation in fed-batch glycoprotein production. Mol Biotechnol. 2012;51(3):272-82.

26. Kottgen E, Hell B, Muller C, Tauber R. Demonstration of glycosylation variants of human fibrinogen, using the new technique of glycoprotein lectin immunosorbent assay (GLIA). Biol Chem Hoppe Seyler. 1988;369(10):1157-66.

27. Patwa TH, Zhao J, Anderson MA, Simeone DM, Lubman DM. Screening of glycosylation patterns in serum using natural glycoprotein microarrays and multi-lectin fluorescence detection. Anal Chem. 2006;78(18):6411-21.

28. Zhou Y, Lu K, Pfefferle S, Bertram S, Glowacka I, Drosten C, et al. A single asparagine-linked glycosylation site of the severe acute respiratory syndrome coronavirus spike glycoprotein facilitates inhibition by mannose-binding lectin through multiple mechanisms. J Virol. 2010;84(17):8753-64.

29. Okamoto K, Takada K, Ikeda R, Aiyama S. Changes in the properties of secretory granules in the palatine gland acinar cells of the postnatally developing rat. Okajimas Folia Anat Jpn. 2008;85(2):49-56.

30. Green DR, Embery G. Isolation, chemical and biological characterization of sulphated glycoproteins synthesized by rat buccal and palatal minor salivary glands in vivo and in vitro. Arch Oral Biol. 1987;32(6):391-9.

31. Samar ME, Avila RE, De Fabro SP, Porfirio V, Esteban FJ, Pedrosa JA, et al. Histochemical study of magellanic penguin (Spheniscus magellanicus) minor salivary glands during postnatal growth. Anat Rec. 1999;254(2):298-306.

32. Samar ME, Avila RE, Esteban FJ, Olmedo L, Dettin L, Massone A, et al. Histochemical and ultrastructural study of the chicken salivary palatine glands. Acta Histochem. 2002;104 (2):199-207.

33. Hakami Z, Kitaura H, Honma S, Wakisaka S, Takano-Yamamoto T. Lectin histochemistry of palatine glands in the developing rat. Acta Histochemica. 2014;116(4):596-605.

34. Monsigny M, Roche AC, Sene C, Maget-Dana R, Delmotte F. Sugar-lectin interactions: how does wheat-germ agglutinin bind sialoglycoconjugates? FEBS. 1980;104(1):147-53.

35. Adi MM, Chisholm DM, Waterhouse JP. Histochemical study of lectin binding in the human fetal minor salivary glands. J Oral Pathol Med. 1995;24(3):130-5.

36. Sato M, Yonezawa S, Uehara H, Arita Y, Sato E, Muramatsu T. Differential distribution of receptors for two fucose-binding lectins in embryos and adult tissues of the mouse. Differentiation. 1986;30(3):211-9.

37. Redman RS, Sreebny LM. Morphologic and biochemical observations on the development of the rat parotid gland. Dev Biol. 1971;25(2):248-79.

38. Jacoby F, Leeson CR. The postnatal development of the rat submaxillary gland. J Anat. 1959;93(2):201-16.

39. Tabak LA. In defense of the oral cavity: structure, biosynthesis, and function of salivary mucins. Annu Rev Physiol. 1995;57:547-64.

40. Klein PB, Weilemann WA, Schroeder HE. Structure of the soft palate and composition of the oral mucous membrane in monkeys. Anat Embryol. 1979;156(2):197-215.

41. Lambert R, Pansu D, Berard A, Vitani C, Dechelette MA. Histochemical studies on human mucous secreting glands in the soft palate, uvula and esophagus. Digestion. 1973;8(2):110-9. 
42. Yonezawa S, Nakamura T, Tanaka S, Sato E. Glycoconjugate with Ulex europaeus agglutininI-binding sites in normal mucosa, adenoma, and carcinoma of the human large bowel. J Natl Cancer Inst. 1982;69(4):777-85.

43. Tandler B. Structure of mucous cells in salivary glands. Microsc Res Tech. 1993;26(1):49-56.

44. Tabak LA, Levine MJ, Mandel ID, Ellison SA. Role of salivary mucins in the protection of the oral cavity. J Oral Pathol. 1982;11(1):1-17.

45. Amerongen AV, Bolscher JG, Veerman EC. Salivary mucins: protective functions in relation to their diversity. Glycobiology. 1995;5(8):733-40.

46. Triantafyllou A, Fletcher D, Scott J. Glycosylations in demilunar and central acinar cells of the submandibular salivary gland of ferret investigated by lectin histochemistry. Arch Oral Biol. 2004;49(9):697-703. 